Diabetologe 2010 - 6:85

DOI 10.1007/s11428-010-0544-4

(c) Springer-Verlag 2010

\title{
T. Haak
}

Diabetes-Zentrum Mergentheim, Diabetes Klinik Bad Mergentheim GmbH \& Co. KG, Bad Mergentheim

\section{Diabetesversorgung in Deutschland}

nun nicht mehr an die RisikostrukturAusgleichsverordnung (RSAV) gebunden sind, stellt sich natürlich die Frage, wohin die DMP sich entwickeln werden und wie deren Zukunft aussieht.

Verändern werden sich auch die Versorgungsformen im ambulanten Bereich. Aufgrund des hohen Drucks durch die Vielzahl von Diabeteskranken wird die Diabetesbehandlung beim Hausarzt einen ganz neuen Stellenwert bekommen. Ähnlich wie vor etwa 10 Jahren stationäre Leistungen in den ambulanten Bereich verlagert wurden, werden nun Leistungen einer Diabetes-Schwerpunktpraxis in den hausärztlichen Bereich verlagert - und dies ist auch gut so. Denn die Kompetenz der Schwerpunktpraxen sollte vor allen Dingen den Patienten vorbehalten bleiben, die diese fachärztliche Kompetenz auch benötigen. Darüber hinaus haben die DMP im Hausarztsektor zu einem enormen Wissenszuwachs geführt, so dass viele Aufgaben, die früher in Schwerpunktpraxen erledigt wurden, auch auf Hausarztebene möglich sein werden.

Überhaupt bedeutet die Diabetesbehandlung stets Teamarbeit, und es geht nicht darum, zu definieren, wer was macht, sondern wie die zu erledigenden Aufgaben bestmöglich gemeinsam erfüllt werden können. Eine besondere Rolle spielt hierbei die integrierte Versorgung, die sich bei der Behandlung des Diabetes und dessen Folgeerkrankungen gerade$\mathrm{zu}$ anbietet.

Alle Aktivitäten auf dem Sektor der Diabetologie benötigen neben einer medizinisch-wissenschaftlichen auch eine öffentlichkeitswirksame Vertretung. Diese gab es bis 2009 nur in sehr beschränktem Umfang, und dies die Wahrnehmung der deutschen Diabetologie kaum gefördert. Doch aus Fehlern kann man lernen, und dies ist geschehen, indem im Jahr 2009 diabetesDE gegründet wurde. In dieser neuen Gesamtorganisation finden alle Menschen, die von Diabetes in irgend einer Weise betroffen oder beruflich damit befasst sind, kompetente Ansprechpartner, Hilfe und eine gemeinsame Vertretung nach außen. Unter diabetesDE kommen Ärzte, Wissenschaftler, Patienten, Berater und all jene zusammen, die diese starke und kompetente Vertretung nach außen benötigen. Im Januar dieses Jahres ist es gelungen, auch die Selbsthilfeorganisation Deutscher Diabetiker Bund (DDB) mit ins Boot zu holen. So gesehen deuten alle Zeichen auf eine gute Entwicklung der deutschen Diabetologie in den kommenden Jahren. Und dies freut mich ganz besonders.

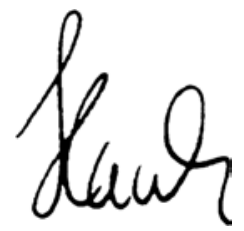

Thomas Haak

\section{Korrespondenzadresse Prof. Dr. T. Haak}

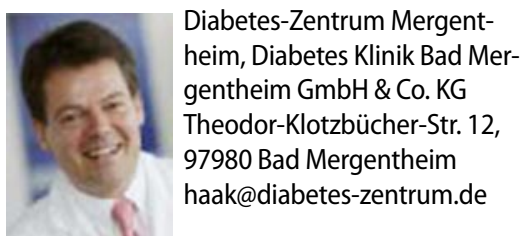

einer permanenten Sorge um die Existenz von Praxen beendet. Nachdem die DMP 